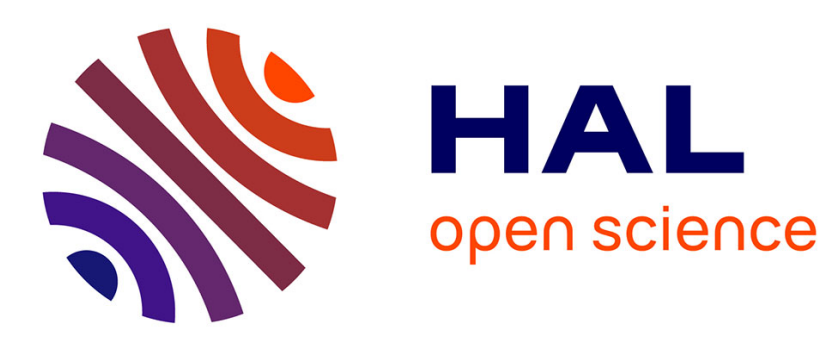

\title{
Self-induced nonlinear spin-orbit interaction of light in liquid crystals
}

\author{
Mohamed El Ketara, Etienne Brasselet
}

\section{To cite this version:}

Mohamed El Ketara, Etienne Brasselet. Self-induced nonlinear spin-orbit interaction of light in liquid crystals. Optics Letters, 2012, 37 (4), pp.602-604. 10.1364/OL.37.000602 . hal-00678838

\section{HAL Id: hal-00678838 \\ https://hal.science/hal-00678838}

Submitted on 14 Mar 2012

HAL is a multi-disciplinary open access archive for the deposit and dissemination of scientific research documents, whether they are published or not. The documents may come from teaching and research institutions in France or abroad, or from public or private research centers.
L'archive ouverte pluridisciplinaire HAL, est destinée au dépôt et à la diffusion de documents scientifiques de niveau recherche, publiés ou non, émanant des établissements d'enseignement et de recherche français ou étrangers, des laboratoires publics ou privés. 


\title{
Self-induced nonlinear spin-orbit interaction of light in liquid crystals
}

\author{
Mohamed El Ketara ${ }^{1,2}$ and Etienne Brasselet ${ }^{1,2, *}$ \\ Univ. Bordeaux, Laboratoire Ondes et Matière d'Aquitaine (LOMA), UMR 5798, F-33400 Talence, France \\ CNRS, LOMA, UMR 5798, F-33400 Talence, France \\ ${ }^{*}$ Corresponding author: e.brasselet@loma.u-bordeaux1.fr
}

Received December 2, 2011; accepted December 15, 2011;

posted December 19, 2011 (Doc. ID 159273); published February 9, 2012

\begin{abstract}
We report on the observation of self-induced nonlinear spin-orbit interaction of light driven by Kerr orientational optical nonlinearities in liquid crystals. It consists of the self-induced spin-to-orbital nonlinear conversion for the angular momentum of light. The optical angular momentum conversion is driven by the creation of a topological liquid crystal defect by the light itself. Moreover, we show that such a nonlinear process can be significantly enhanced by using additional electric fields. (c) 2012 Optical Society of America

OCIS codes: $\quad 260.6042,160.3710,190.4720,160.1245$.
\end{abstract}

The coupling between the spin of a particle and its motion, the spin-orbit interaction, is the underlying mechanism of many fundamental phenomena. For photons, it can manifest as the spin Hall [1] or the orbital Hall [2] effect. It may also lead to the conversion between the spin and orbital parts of the photon angular momentum [3]. Up to now, spin-to-orbital optical angular momentum conversion (STOC) has been thoroughly studied, however, almost exclusively in the linear regime, where the process is independent of the light intensity.

In the linear regime, the efficiency of the STOC process, defined as the fraction of the incident power converted into a vortex beam, is completely determined by the material properties and the light-matter interaction geometry. In contrast, in the nonlinear regime, the STOC efficiency depends on the incident light beam power too-a barely studied situation. Still, one can mention a few recent works: thermally induced nonlinear STOC in isotropic crystals [4] and spin-orbit optical crossphase-modulation in liquid crystals [5]. Nevertheless, the observation of a self-induced nonlinear STOC via a nonlinear optical process has not been reported so far, to the best of our knowledge.

Here we report on the observation of a self-induced nonlinear spin-orbit interaction of light in nematic liquid crystals. The main idea relies on the fact that a liquid crystal topological defect can behave as a spin-orbit converter for the photon angular momentum, as previously shown in nematic droplets [6] and films [7]. In the present case, the light itself creates a topological defect via a nonlinear topological optical reorientation lossless process [8]. In turn, the generated defect is responsible for an additional conversion of part of the incoming light into an optical vortex carrying orbital angular momentum. This situation is illustrated in Fig. 1. Such a nonlinear STOC process is also shown to be strongly enhanced using low-voltage external electric fields.

In our experiments, the nematic film is prepared to have its director $\mathbf{n}$ (a unit vector that represents the local average orientation of the rodlike molecules of the nematic liquid crystal), hence its optical axis, perpendicular to the cell walls owing to strong anchoring boundary conditions. In the linear regime, which corresponds to the limit of low laser beam power, the nematic film thus be-

haves as a $c$-cut uniaxial solid crystal slab; see Fig. 1 (a). Such an optical element is known to partially convert a normally incident circularly polarized Gaussian beam into a contracircularly polarized vortex beam endowed with a topological charge two-phase singularity $[\underline{9}, \underline{10}]$. This is illustrated in Fig. 1(a), where $\mathbf{e}_{\sigma}=\left(\mathbf{e}_{x}+\right.$ $\left.i \sigma \mathbf{e}_{y}\right) / \sqrt{2}$ refers to the circular polarization basis with $\left(\mathbf{e}_{x}, \mathbf{e}_{y}, \mathbf{e}_{z}\right)$ as the Cartesian coordinate system (the light propagates along the $z$ axis) and $\sigma= \pm 1$ for the leftand right-handed circular polarization states. In Fig. 1(a), the phase factor $e^{2 i \sigma \phi}$ refers to the generated phase singularity with topological charge $2 \sigma$ reminiscent of the conservation of the total angular momentum of light, which results from the axial symmetry of the light-matter system.

The linear STOC efficiency associated to a film of thickness $L$ expresses as $\eta_{0}=\frac{1}{2}\left(L / L_{c}\right)^{2} /\left[1+\left(L / L_{c}\right)^{2}\right]$ [10], where $L_{c}=\lambda\left(2 n_{\|}^{2} /\left[\pi \tan ^{2} \theta_{0} n_{\perp}\left(n_{\|}^{2}-n_{\perp}^{2}\right)\right]\right)$ is a characteristic anisotropic diffraction length, $\lambda$ is the wavelength, $n_{\| . \perp}$ are the refractive indices along and perpendicular to $\mathbf{n}$, and $\theta_{0}$ is the Gaussian beam divergence angle inside the material. The previous expression holds for moderate focusing, i.e., when the paraxial approximation is justified, which is the case in all our experiments, as demonstrated in Fig. 2(a).

(a) Linear regime

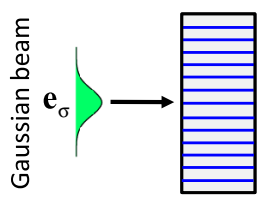

Nematic at rest

$$
\eta=\eta_{0}
$$

(b) Nonlinear regime

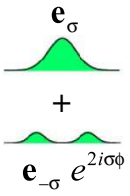

Light-induced topological defect

Fig. 1. (Color online) Illustration of our experiment. A circularly polarized Gaussian beam impinges at normal incidence onto a nematic film with perpendicular alignment at rest; see panel (a), where lines refer to the director field. (a) The linear STOC is characterized by the conversion efficiency $\eta=\eta_{0}$. (b) When a topological defect is created by light, the vortex contribution to the output field increases. This leads to a nonlinear STOC efficiency of the form $\eta=\eta_{0}+\Delta \eta$. 

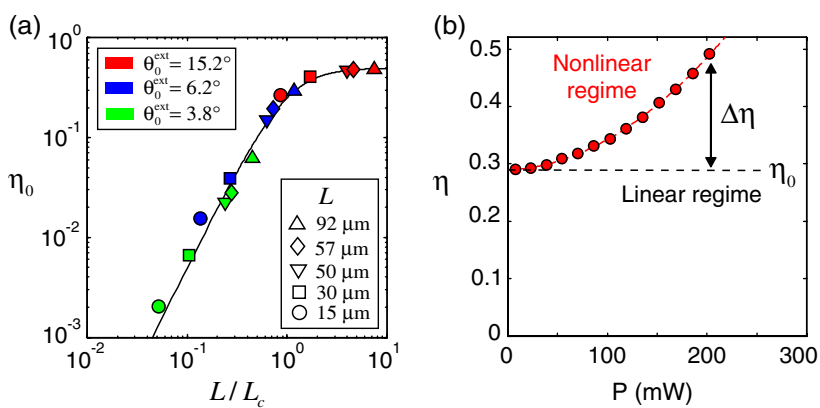

Fig. 2. (Color online) (a) STOC efficiency in the linear regime, $\eta_{0}$, versus reduced thickness $L / L_{c}$ for MLC-2079 ( $L=15$ and $30 \mu \mathrm{m})$ and E7 $(L=50,57$, and $92 \mu \mathrm{m})$ nematic films. The external beam divergence angle is either $\theta_{0}^{\text {ext }}=3.8^{\circ}, 6.2^{\circ}$, or $15.2^{\circ}$, with $\theta_{0} \simeq \theta_{0}^{\text {ext }} / n_{\perp}$. The solid curve refers to the universal behavior $\eta_{0}\left(L / L_{c}\right)$, whose expression is given in the text. (b) Illustration of the self-induced nonlinear STOC as a result of the creation of a liquid crystal defect by light for a $92-\mu \mathrm{m}$-thick E7 film located at $\langle z\rangle=400 \mu \mathrm{m}$ and illumination condition such as $\eta_{0}=0.29$.

In practice, $\eta_{0}$ is measured as the ratio between the power carried by output light field component whose circular polarization state is orthogonal to the incident one and the total input beam power $P$. This is achieved by using a quarter-wave plate oriented at $\pm 45^{\circ}$ from a polarizer, depending on the handedness of the input polarization state. In experiments, we used $\lambda=532 \mathrm{~nm}$ and two different commercial materials, $\mathrm{E} 7\left(n_{\perp}=1.52\right.$ and $n_{\|}=$ 1.74 at $589 \mathrm{~nm})$ and MLC-2079 $\left(n_{\perp}=1.49\right.$ and $n_{\|}=1.64$ at $589 \mathrm{~nm}$ ). We explored various cell thicknesses and external (i.e., in the air) divergence angle $\theta_{0}^{\text {ext }}$, which prescribes an internal divergence angle $\theta_{0} \simeq \theta_{0}^{\text {ext }} / n_{\perp}$. Experimental results are shown in Fig. 2(a), which gathers all the data obtained with different materials, beam divergences, and cell thicknesses.

As illustrated in Fig. 2(b), the STOC efficiency increases with $P, \eta=\eta_{0}+\Delta \eta$. Such a behavior is observed (i) whatever is the linear regime efficiency $\eta_{0}$ and (ii) whatever is the distance $\langle z\rangle$ between the incident beam waist location and the middle of the film. Experimental results for a

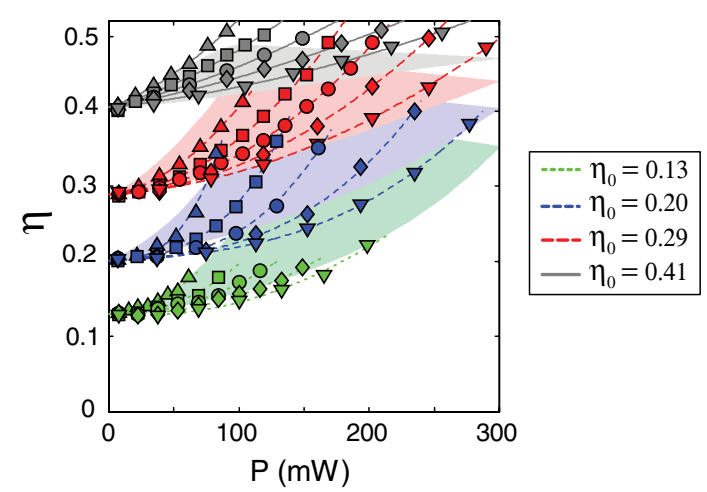

Fig. 3. (Color online) Nonlinear STOC for a $92-\mu$ m-thick E7 nematic film. The measured $\eta$ is plotted as a function of the input beam power $P$ for different values of $\eta_{0}$ and various sample locations $\langle z\rangle=200$ (triangles), 300 (squares), 400 (circles), 500 (diamonds) and $600 \mu \mathrm{m}$ (inverted triangles); curves are guides for the eyes. The shaded regions refer to the values explored by $\eta$ predicted by our topological optical reorientation model for the range of parameters used in experiments.
92- $\mu$ m-thick cell of E7 are shown in Fig. 3 for $\eta_{0}=$ $(0.13,0.2,0.29,0.41)$, which correspond to beam divergence angles $\theta_{0}=\left(4.3^{\circ}, 5.1^{\circ}, 6^{\circ}, 8^{\circ}\right)$, and various sample locations from $\langle z\rangle=200$ to $600 \mu \mathrm{m}$ by $50 \mu \mathrm{m}$ steps (the data shown in Fig. 3 are restricted to a $100 \mu \mathrm{m}$ step for better readability). Although all these data refer to input beam power values for which the axial symmetry is preserved, the latter symmetry is spontaneously broken above a critical power $P=P_{c}$, found to depend on $\eta_{0}$ and $\langle z\rangle$. Above $P_{c}$, a dynamical regime with a nonaxisymmetric distorted director field may take place but its study is beyond the scope of the present work.

To understand these observations, let us recall that a circularly polarized Gaussian beam impinging at normal incidence onto a perpendicularly aligned nematic film can generate axially symmetric topological defects with topological charge one even under moderate focusing conditions [8]. Namely, the director defines an inhomogeneous anisotropy that exhibits a defect around which the director orientation winds by $2 \pi$. This extends the concept of space-variant anisotropic plates with uniform birefringence [11], which are known for their ability to realize STOC. Indeed, here the birefringence varies along the radial coordinate as a result of a doughnut-shaped light-induced distorted director profile [8]. We therefore expect any light-induced defect to modify the spin-orbit interaction of light, hence the STOC efficiency.

Still, one could rightly argue that thermal effects represent another possible source of STOC efficiency changes via the temperature dependence of the material parameters. To gauge the influence of thermal effects on STOC, we shall restrict ourselves to the linear regime. This implies a need to evaluate the dependence of $L_{c}$ on the temperature $T$. From [12], we calculate $\left(\partial L_{c} / \partial T\right) / L_{c}=$ $5 \times 10^{-3} \mathrm{~K}^{-1}$ at room temperature with $\lambda=589 \mathrm{~nm}$, the sign of the latter quantity being positive, whatever is $T$. Considering solely thermal effects we, therefore, expect $\Delta \eta<0$, whereas we observe $\Delta \eta>0$. This supports our interpretation based on topological optical reorientation.

Also, we note that all data reported here correspond to $P<P_{c} \lesssim P_{F}$, where $P_{F}$ is the so-called optical Fréedericksz transition threshold power above which an orientational instability is expected to take place for a perpendicularly aligned nematic film illuminated at normal incidence [13]. This also agrees with our interpretation, since the light-induced generation of a liquid crystal defect is a thresholdless phenomenon [8]. Indeed, we find $P_{c} / P_{F}=0.48 \pm 0.2$ over the 45 different sets of parameters $\left\{\eta_{0},\langle z\rangle\right\}$ that have been used during the experiments (not all of them are shown in Fig. 3). The latter ratio is obtained from the measured value of $P_{c}$ and the analytical expression in the case of circularly polarized incident light field, $P_{F}=\pi^{3}(\langle w\rangle+L \sqrt{2} /$ $\pi)^{2} c K_{3} n_{\|}^{2} /\left[L^{2} n_{\perp}\left(n_{\|}^{2}-n_{\perp}^{2}\right)\right][\underline{14}]$, where $\langle w\rangle$ is the beam radius at $\exp (-2)$ of its maximum intensity in the middle of the film, $c$ is the speed of light, and $K_{3}$ is the bend elastic constant (taking $K_{3}=17 \mathrm{pN}$ for E7, the latter expression gives the range of values $P_{F} \approx 0.2-1 \mathrm{~W}$ for our experiments).

A quantitative description of our observations is achieved by exploiting the model of topological optical reorientation developed in [15] in the case of a beam waist located at the input facet of the film and neglecting 

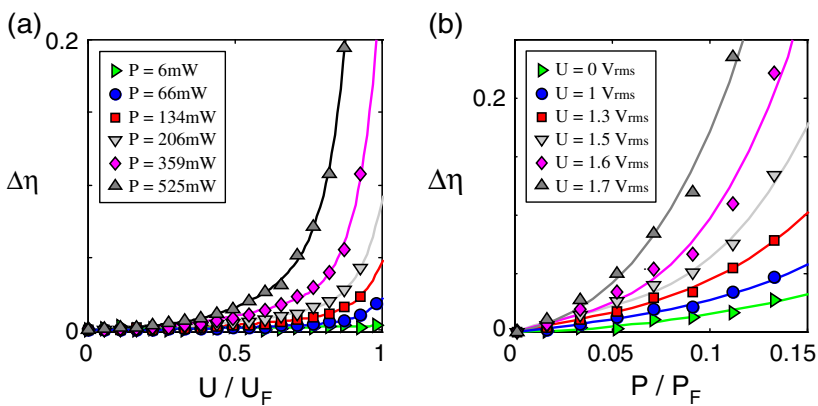

Fig. 4. (Color online) Electric field enhancement of the nonlinear STOC efficiency. (a) $\Delta \eta$ versus the reduced applied voltage $U / U_{F}$ at fixed input beam power. (b) $\Delta \eta$ versus the reduced power $P / P_{F}$ at fixed applied voltage. Curves are guides for the eyes. The data correspond to a MLC-2079 nematic film with thickness $L=30 \mu \mathrm{m}$ under illumination conditions such as $\langle w\rangle=60 \mu \mathrm{m}$ and $\eta_{0}=0.41$.

the elastic anisotropy of the nematic. The model is here extended to any value of $\langle z\rangle$, also accounting for elastic anisotropy, but hereafter we skip the details of calculations. First, we determine the effective local polar reorientation angle $\delta \theta(r, z)=\arcsin \left[\left|\mathbf{n}(r, z) \times \mathbf{e}_{z}\right|\right]$ associated to the light-induced defect. Then, it is possible to deduce $\eta$ in the nonlinear regime from its geometrical-optics expression in the linear regime $\eta_{0}=\int_{0}^{\pi / 2} f(\theta) \sin ^{2}\left[\psi_{0}(\theta) /\right.$ $2] \mathrm{d} \theta / \int_{0}^{\pi / 2} f(\theta) \mathrm{d} \theta$, where $f(\theta)=\left(\tan \theta / \cos ^{2} \theta\right) \times \exp$ $\left(-2 \tan ^{2} \theta / \tan ^{2} \theta_{0}\right)$ and $\psi_{0}(\theta)=(\pi L / \lambda)\left(n_{\perp} / n_{\|}^{2}\right)\left(n_{\|}^{2}-n_{\perp}^{2}\right)$ $\tan ^{2} \theta$. Indeed the term $\sin ^{2}\left[\psi_{0}(\theta) / 2\right]$ can be interpreted as the STOC efficiency associated to the partial wave associated with the optical ray making an angle $\theta$ with the $z$ axis. Therefore, we evaluate $\eta$ from the latter expression of $\eta_{0}$ by performing the change $L \tan ^{2} \theta \rightarrow \int_{z_{\text {in }}}^{z_{1}}+L \tan ^{2}[\theta+$ $\delta \theta(r=z \tan \theta, z)] \mathrm{d} z$ into the expression of the phase factor $\psi_{0}(\theta), z_{\text {in }}=\langle z\rangle-L / 2$ being the location of the film input facet.

Results are shown in Fig. $\underline{3}$, where, for each value of $\eta_{0}$, the shaded area corresponds to the predicted values explored by $\eta$ for $200 \leq\langle z\rangle \leq 600 \mu \mathrm{m}$, as used in experiments. Moreover, in order to emphasize the selfconsistency of the model with respect to the small reorientation amplitude approximation [15], we restricted the simulations to $\delta \theta<0.05$. Noting that no adjustable parameter is used in the model, we conclude to a satisfying agreement.

The observed STOC for the photon angular momentum can be further enhanced using additional electric fields. This is demonstrated by using a $30-\mu \mathrm{m}$-thick nematic cell provided with transparent electrodes and filled with MLC-2079, whose anisotropy of the relative dielectric permittivity is negative at low frequency, namely, $\varepsilon_{a}=$
-6.1 at $1 \mathrm{kHz}$. Therefore, at $P=0$, the director remains at rest up to the electrical Fréedericksz transition threshold characterized by an applied voltage $U=U_{F}$ (in our case, $U_{F}=1.83 V_{\text {rms }}$ ). In contrast, when $P \neq 0$, the topological optical reorientation is present and is, in turn, electrically assisted even if $U<U_{F}$. Consequently, the nonlinear STOC is electrically enhanced. This is shown in Fig. 4(a), when $U$ is varied at fixed $P$, and in Fig. 4(b), when $P$ is varied at fixed $U$.

Finally, to gauge how efficient is the observed selfinduced STOC, we define $\xi=\Delta \eta / P$ as the figure of merit of the nonlinear process, where $\Delta \eta$ is the typical variation of $\eta$ under laser beam power $P$. Here we found $\xi \sim$ $1 \mathrm{~W}^{-1}$ with $\Delta \eta \sim 0.1$ and $P \sim 100 \mathrm{~mW}$; see Fig. 3 . This is more than 5 orders of magnitude larger than previously reported nonlinear STOC mediated by thermal effects in isotropic crystals where $\xi \sim 5 \times 10^{-6} \mathrm{~W}^{-1}$ with $\Delta \eta \sim 5 \times$ $10^{-4}$ and $P \sim 100 \mathrm{~W}[\underline{4}]$.

To conclude, we reported on the possibility to significantly modify the orbital angular momentum of a light beam through a self-induced nonlinear optical lossless process. The demonstration has been made using low power cw laser sources, and we showed that the effect can be significantly electrically enhanced using lowfrequency low-amplitude applied voltages.

\section{References}

1. M. Onoda, S. Murakami, and N. Nagaosa, Phys. Rev. Lett. 93, 083901 (2004).

2. K. Y. Bliokh, Phys. Rev. Lett. 97, 043901 (2006).

3. K. Y. Bliokh, M. A. Alonso, E. A. Ostrovskaya, and A. Aiello, Phys. Rev. A 82, 063825 (2010).

4. S. Mosca, B. Canuel, E. Karimi, B. Piccirillo, L. Marrucci, R. D. Rosa, E. Genin, L. Milano, and E. Santamato, Phys. Rev. A 82, 043806 (2010).

5. E. Brasselet, Phys. Rev. A 82, 063836 (2010).

6. E. Brasselet, N. Murazawa, H. Misawa, and S. Juodkazis, Phys. Rev. Lett. 103, 103903 (2009).

7. E. Brasselet and C. Loussert, Opt. Lett. 36, 719 (2011).

8. E. Brasselet, Opt. Lett. 34, 3229 (2009).

9. A. Volyar and T. Fadeyeva, Opt. Spectrosc. 94, 235 (2003).

10. A. Ciattoni, G. Cincotti, and C. Palma, J. Opt. Soc. Am. A 20, 163 (2003).

11. G. Biener, A. Niv, V. Kleiner, and E. Hasman, Opt. Lett. 27, 1875 (2002).

12. J. Li, S.-T. Wu, S. Brugioni, R. Meucci, and S. Faetti, J. Appl. Phys. 97, 073501 (2005).

13. N. V. Tabiryan, A. V. Sukhov, and B. Y. Zel'dovich, Mol. Cryst. Liq. Cryst. 136, 1 (1986).

14. E. Brasselet, A. Lherbier, and L. J. Dubé, J. Opt. Soc. Am. B 23, 36 (2006).

15. E. Brasselet, J. Opt. 12, 124005 (2010). 